\title{
Nonlinear Control of a Flexible Joint Robotic Manipulator with Experimental Validation
}

\author{
Waqar Alam ${ }^{1}$ - Adeel Mehmood ${ }^{1}$ - Khurram Ali ${ }^{1}$ - Usman Javaid ${ }^{1}$ - Soltan Alharbi ${ }^{2}$ - Jamshed Iqbal2,3,* \\ 1 COMSATS Institute of Information Technology, Department of Electrical Engineering, Pakistan \\ 2 University of Jeddah, Electrical and Computer Engineering Department, Saudi Arabia \\ 3 FAST National University of Computer and Emerging Sciences, Department of Electrical Engineering, Pakistan
}

This article addresses the design and implementation of robust nonlinear control approaches to obtain the desired trajectory tracking of a flexible joint manipulator driven with a direct-current (DC) geared motor. The nonlinear control schemes have been designed and implemented such that they locally stabilize the closed loop system considering all the states as bounded. The system model has been derived using EulerLagrange approach. Two different approaches based on sliding mode control (SMC), i.e. the traditional SMC and integral SMC, have been considered in the present study. To experimentally validate the proposed control laws, an electrically-driven single-link flexible manipulator has been designed and fabricated. The designed control algorithms have been developed and experimentally validated on the custom-developed platform. The results obtained both from MATLAB/Simulink and the experimental platform verify the performance of the proposed control algorithms.

Keywords: flexible joint manipulator; modern control system; sliding mode control; integral sliding mode control

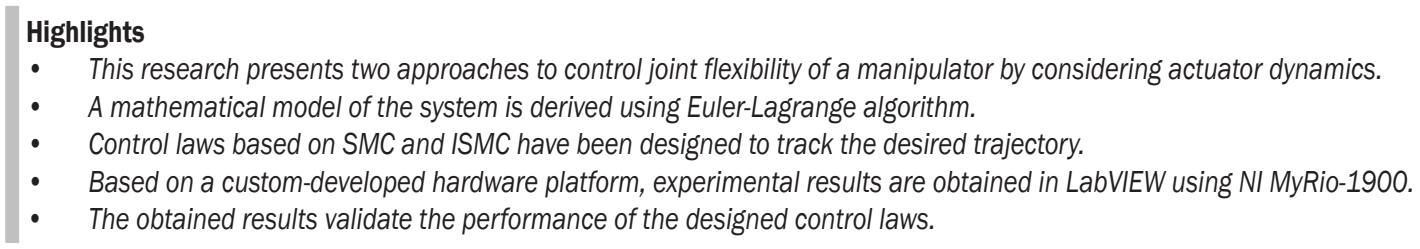

\section{INTRODUCTION}

The domain of robotics has recently drawn significant attention in the scientific community [1]. In industrial applications, trajectory tracking control [2] of flexible joint manipulators has received considerable attention in the last two decades. An accurate end effector's position through reliable control approaches is critical for high-performance robotic applications accomplishing dangerous and tedious jobs [3]. Thus, a robotic manipulator is designed so as to increase stiffness in order to reduce undesirable oscillations of the end effector to track desired positions. The stiffness can be achieved by using heavy materials, which may increase power consumption and decrease the speed of operation [4]. The basic approach to maximize operational speed and minimize power consumption is to use lightweight, flexible joint manipulators; however, this is subject to improving performance in endpoint tracking.

In [5], it is emphasized, that joint flexibility and actuator dynamics should be considered while modelling as well as designing an appropriate control law so as to achieve high performance. The literature focused on the design of controllers for flexible joint manipulators is extensive. However, incorporating actuator dynamics in the modern control of the end effector's position of a flexible joint manipulator remains a challenging issue in the robotics community [6]. Some methodologies are reported to control the manipulator without taking into account the dynamics of the actuator. Examples include feedback linearization method [7], the singular perturbation approach [8], the integral manifold control [9], the passivity approach [10], the proportional derivative (PD) control approach [7] and the adaptive sliding mode technique [11]. The major limitation of the above-mentioned research works is that these schemes assumed torque to be an input to the rigid link. However, it is highlighted in [4] that the actuator dynamics are of great importance in electro-mechanical systems, especially in a case in which a system has to deal with varying loads. Ailon and Lozano [12] proposed an iterative control law to regulate the set-point of a flexible robotic system that is driven electrically and is subjected to model uncertainty. Another study reported [13] presents a controller based on adaptation law for a flexible joint robot to improve the trajectory tracking in the presence of time-variable uncertainty in the system's parameters. A traditional back-stepping approach has been applied successfully to solve the control problem of robots with flexible joints [14] to [16]. However, in these approaching, the torque is assumed 
to be directly applied to the links of the robot. In [16], a robust control law based on a back-stepping technique for tracking trajectory of manipulators has been proposed, in which only armature current and link position are measured for feedback purposes. Despite these efforts [14] to [16], most of the reported back-stepping techniques suffer from two limitations; The first is that the systems under consideration did not have time-varying parametric uncertainties. The second limitation is related to the level of complexity resulting from iterative differentiations of nonlinear virtual functions and thus leading to a complex and computationally expensive algorithm.

In the present article, the problem of robust control law design for accurate trajectory tracking of a flexible joint manipulator is addressed by considering actuator dynamics, joint flexibility, and viscous friction. Owing to computational simplicity and ease of implementation, the nonlinear law has been realized on custom-developed hardware. The control input (i.e. output of the controller) is fed to the plant (i.e. the direct-current (DC) motor and the flexible joint).

\section{MATHEMATICAL MODELLING}

To derive the mathematical model of the system, the considered parameters and their values are listed in Table 1.

Table 1. Description of system parameters

\begin{tabular}{clccc}
\hline S\# & Parameter & Symbol & Value & Unit \\
\hline 1 & Link mass & $\mathrm{m}$ & 1 & $\mathrm{~kg}$ \\
\hline 2 & Gears ratio & $\mathrm{N}$ & 1 & - \\
\hline 3 & Armature resistance & $\mathrm{R}$ & 1.6 & $\Omega$ \\
\hline 4 & Joint stiffness & $\mathrm{k}$ & 14 & $\mathrm{~N} \cdot \mathrm{m} / \mathrm{rad}$ \\
\hline 5 & Motor torque constant & $\mathrm{kt}$ & 0.2 & $\mathrm{~N} \cdot \mathrm{m} / \mathrm{A}$ \\
\hline 6 & Back EMF constant & $\mathrm{B}$ & 0.001 & $\mathrm{~N} \cdot \mathrm{m} \cdot \mathrm{s} / \mathrm{rad}$ \\
\hline 7 & Link length & $\mathrm{d}$ & 0.5 & $\mathrm{M}$ \\
\hline 8 & Gravitational acceleration & $\mathrm{g}$ & 10 & $\mathrm{~m} / \mathrm{s}^{2}$ \\
\hline 9 & Link moment of inertia & $\mathrm{J} 1$ & 1 & $\mathrm{~kg} \cdot \mathrm{m}^{2}$ \\
\hline 10 & Motor shaft inertia & $\mathrm{J} 2$ & 0.3 & $\mathrm{~kg} \cdot \mathrm{m}^{2}$ \\
\hline 11 & Armature inductance & $\mathrm{L}$ & 0.01 & $\mathrm{H}$ \\
\hline & & & &
\end{tabular}

The flexibility in joint is modelled as a linear torsional spring [16]. Using Euler Lagrange equation, the equations of motion for an electro-mechanical system can be derived. The total kinetic energy (PE) can be written as Eqs. (1) and (2):

$$
\begin{gathered}
K E=\frac{1}{2} J_{1} \dot{q}_{1}^{2}+\frac{1}{2} J_{2} \dot{q}_{2}^{2}, \\
P E=m g d\left(1-\cos q_{1}\right)+\frac{1}{2} k\left(q_{1}-q_{2}\right)^{2},
\end{gathered}
$$

where $q_{1}$ and $q_{2}$ are angular positions of the link and the motor shaft respectively, while $J_{1}$ and $J_{2}$ are coefficients corresponding to link and motor inertia respectively. The Lagrange equation is:

$$
L=K E-P E \text {. }
$$

Using Eqs. (1) and (2), Eq. (3) can be written as:

$L=\frac{1}{2} J_{1} \dot{q}_{1}+\frac{1}{2} J_{2} \dot{q}_{2}-m g d\left(1-\cos q_{1}\right)+\frac{1}{2} k\left(q_{1}-q_{2}\right)^{2}$.

Lagrangian equations of motion with respect to motor and link angular position can be written as:

$$
\begin{gathered}
\frac{d}{d t} \frac{\partial L}{\partial \dot{q}_{2}}-\frac{\partial L}{\partial q_{1}}=0, \\
\frac{d}{d t} \frac{\partial L}{\partial \dot{q}_{2}}-\frac{\partial L}{\partial q_{2}}=\tau-B \dot{q}_{2},
\end{gathered}
$$

while the torque produced is:

$$
\tau=k_{t} I
$$

where is the armature current. Now taking derivatives of Eqs. (5) and (6):

$$
\begin{gathered}
\frac{\partial L}{\partial \dot{q}_{1}}=J_{1} \dot{q}_{1}, \quad \frac{\partial L}{\partial \dot{q}_{2}}=J_{2} \dot{q}_{2}, \\
\frac{d}{d t} \frac{\partial L}{\partial \dot{q}_{1}}=J_{1} \ddot{q}_{1}, \quad \frac{d}{d t} \frac{\partial L}{\partial \dot{q}_{2}}=J_{2} \ddot{q}_{2} . \\
\frac{\partial L}{\partial q_{1}}=-M g d\left(1-\sin \left(q_{1}\right)\right)-k\left(q_{1}-q_{2}\right), \\
\frac{\partial L}{\partial q_{1}}=k\left(q_{1}-q_{2}\right) .
\end{gathered}
$$

Equations of motion for the mechanical subsystem are given as:

$$
\begin{gathered}
J_{1} \ddot{q}_{1}+m g d \sin \left(q_{1}\right)+k\left(q_{1}-q_{2}\right), \\
J_{2} \ddot{q}_{2}-k\left(q_{1}-q_{2}\right)=\tau-B \dot{q}_{2}
\end{gathered}
$$

The flexible joint manipulator consists of a DC gear motor whose equation is derived by applying Kirchhoff's voltage law (KVL):

$$
V=V_{R}+V_{L}+e,
$$

where $V_{R}$ is the voltage across resistor, $V_{L}$ is the voltage across inductor and $e$ is back electro motive force (emf) given by:

$$
e=K_{b} \dot{q}_{2},
$$

where $K_{b}$ is back emf constant. In this system, the input voltage to the DC motor is the excitation input given as: 


$$
v=u=R I+L \frac{d i}{d t}+K_{b} \dot{q}_{2},
$$

where $u$ is the motor input voltage. The nonlinear dynamics of the system can be formulated as:

$$
\begin{gathered}
J_{1} \ddot{q}_{1}+m g d \sin \left(q_{1}\right)+k\left(q_{1}-q_{2}\right), \\
J_{2} \ddot{q}_{2}-k\left(q_{1}-q_{2}\right)+B \dot{q}_{2}=k_{t} I .
\end{gathered}
$$

Assumption 1: The parameters mentioned in Eq. (17) can be expressed as $R()=.R_{0}+\Delta R(t)$, $L()=.L_{0}+\Delta L(t)$, and $K_{b}()=.K_{b 0}+\Delta K_{b}(t)$.

Assumption 2: The parameters mentioned in Eq. (16) can be expressed as $J_{2}()=.J_{20}+\Delta J_{2}(\mathrm{t})$, $B()=.B_{0}+\Delta B(t)$, and $K_{t}()=.K_{t 0}+\Delta K_{t}(t)$.

Remarks: In these realistic assumptions, the system parameters are split into a known nominal value and the unknown part, which is considered to be uncertain. In practical control systems, parameters may not be unknown completely, their nominal values are precisely known to us; this is taken into consideration in this research.

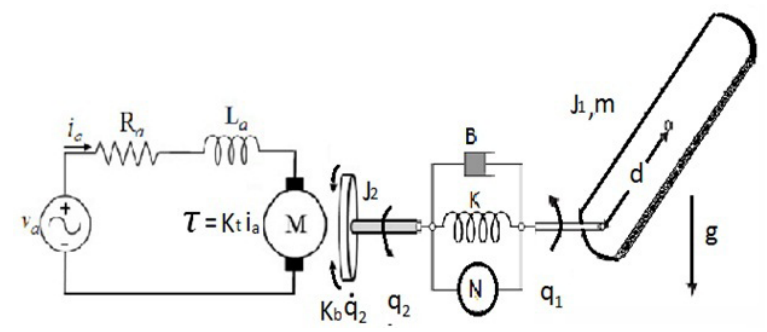

Fig. 1. Schematic diagram of flexible joint manipulator

The equations of the motion of an electrically driven flexible joint manipulator in state space form are represented below. The state vector contains an angular position and angular velocity of the link side, angular position and angular velocity on the motor side, and the motor armature current, i.e. $\left[x_{1}, x_{2}, x_{3}, x_{4}, x_{5}\right]=\left[q_{1}, \dot{q}_{1}, q_{2}, \dot{q}_{2}, I\right]$.

$$
\begin{gathered}
\dot{x}=f(x)+g(x) u, \quad x \in R^{5}, \\
\dot{x}_{1}=x_{2}, \quad \dot{x}_{2}=-a \sin \left(x_{1}\right)-b\left(x_{1}-x_{3}\right), \\
\dot{x}_{3}=x_{4}, \quad \dot{x}_{4}=O\left(x_{1}-x_{3}\right)-\frac{B}{J_{2}} x_{4}+d x_{5}, \\
\dot{x}_{5}=-\frac{R}{L} x_{5}-\frac{k_{b}}{L} x_{4}+\frac{1}{L} u,
\end{gathered}
$$

where $O=\left(k / J_{2}\right), b=\left(k / J_{1}\right), a=\left(m g d / J_{1}\right)$ and $d=\left(k / J_{2}\right)$. Here, the angular position of the link is considered to be an output. The system equations are complex and exhibit highly nonlinear dynamics thereby highlighting the challenge involved in the controller design.

\section{CONTROLLER DESIGN}

The control objective is to accurately control the position of a flexible joint in finite time by control laws. The proposed control laws are based on two robust nonlinear control techniques: sliding mode control (SMC) and integral SMC.

\subsection{Sliding Mode Control Technique}

SMC is a robust nonlinear control scheme, with a powerful capability to reject disturbances and plant uncertainties. This control scheme works on the principle of continuously altering the configuration of the controller to keep the state variables on the sliding manifold. Due to this phenomenon, undesirable chattering occurs. In mechanical parts, this produces high wear, while in electric parts, it may cause high heat losses [17]. In SMC, the controller comprises two phases [18]; one is used to force the system trajectory to achieve a sliding manifold, while second one is used to drive the states on the sliding manifold toward desired equilibrium point. Consider a linear system given in Eq. (20).

$$
\dot{\xi}=A \xi+B u,
$$

where $A$ and $B$ represent coefficients of the system and $\xi$ is the state vector. The sliding manifold for this system can be selected as:

$$
S=c^{T} \xi,
$$

where $c$ is the sliding mode control design parameters matrix. Differentiating Eq. (21), we obtain:

$$
\dot{\dot{S}}=c^{T} \xi=c^{T}(A \xi+B u) \text {. }
$$

Since, $\dot{S}=0$ during sliding mode:

$$
\dot{S}=c^{T}(A \xi+B u)=0 .
$$

In SMC, the control law $u$ is a combination of two types of control given by:

$$
u=u_{e q}+u_{d i s},
$$

where $u_{e q}$ is the equivalent controller used to control the system states during the sliding phase. Eq. (22) is solved to obtain the equivalent control given by:

$$
u_{e q}=\left(c^{T} B\right)^{-1}\left(c^{T} A \xi\right),
$$


while $u_{\text {dis }}$ represents the switching controller that ensures the system stability.

$$
u_{\text {dis }}=-k_{1} \operatorname{sign} S-k_{2} S,
$$

where $k_{1}>0$ and $k_{2}>0$ are the design parameters and:

$$
\operatorname{sign}(S)=\left\{\begin{array}{c}
1 \text { for } S>0 \\
-1 \text { for } S<0
\end{array} .\right.
$$

Substituting Eqs. (25) and (26) in Eq. (24):

$$
u=\left(-k_{1} \operatorname{sign} S-k_{2} S-\left(c^{T} A \xi\right)\right)\left(c^{T} B\right)^{-1} .
$$

This is the control law $u$ which is required for driving the system's initial states to equilibrium point in a finite time. The proposed control method is now applied to a flexible joint manipulator. The sliding surface selected for the system is:

$$
S=\left(\frac{d}{d t}+c\right)^{n-1} e,
$$

where $e=x_{1}-x_{d}$ is the error between the actual and desired outputs. $n$ is the relative degree of the system, $c$ is a constant known as design parameter, i.e. $c>0$.

$$
S=\left(\frac{d}{d t}+c\right)^{2}\left(\frac{d}{d t}+c\right)^{2} e
$$

After expanding Eq. (29):

$$
S=\dddot{e}+4 \dddot{e} c+6 \ddot{e} c^{2}+4 \dot{e} c^{3}+\dot{e} c^{4} .
$$

Taking derivative of error variables, we obtain:

$$
\begin{aligned}
e= & x_{1}-x_{d}, \quad \dot{e}=x_{2}-\dot{x}_{d}, \quad \ddot{e}=\dot{x}_{2}-\ddot{x}_{d}, \\
\dddot{e}= & -a \cos \left(x_{1}\right) \dot{x}_{2}+a\left(x_{2}\right)^{2} \sin \left(x_{1}\right)-b x_{2}+ \\
& +b\left(\dot{O}\left(x_{1}-x_{3}\right)-\left(B / J_{2}\right) x_{4}+d x_{5}\right)+b f_{1}+\dddot{x}_{d} .
\end{aligned}
$$

For the sake of brevity, a symbol $W$ is defined as:

$$
\begin{gathered}
W=c^{4} x_{2}+4 c^{3} \dot{x}_{2}+6 c^{2} \ddot{x}_{2}+4 c\left(-a \cos \left(x_{1}\right) \dot{x}_{2}+\right. \\
a x_{2}^{2} s \sin \left(x_{1}\right)+a b \sin \left(x_{1}\right)+b^{2}\left(x_{1}-x_{3}\right)+ \\
\left.b O\left(x_{1}-x_{3}\right)-\frac{b B}{J_{2}} x_{4}+b d x_{5}\right)-a \cos \left(x_{1}\right) \ddot{x}_{2}+ \\
a x_{2} \dot{x}_{2} \sin \left(x_{1}\right)+2 a x_{2} \dot{x}_{2} \sin \left(x_{1}\right)+a x_{2}^{3} \cos \left(x_{1}\right)+ \\
a b x_{2} \cos \left(x_{1}\right)+b^{2}\left(x_{2}-x_{4}\right)+b O\left(x_{2}-x_{4}\right)- \\
\left.\left(\frac{B b}{J_{2}}\right)\left(O\left(x_{1}-x_{3}\right)\right)-x_{4} \frac{B}{J_{2}}+d x_{5}\right)+ \\
b d\left(\left(\frac{-R}{L}\right) x_{5}-\frac{k_{b}}{L} x_{4}\right)- \\
c^{4} \dot{x}_{d}-4 c^{3} \ddot{x}_{d}+6 c^{2} \dddot{x}_{d}-4 c \dddot{x}_{d}-\dddot{x}_{d} .
\end{gathered}
$$

Substituting and taking derivative of $S$,

$$
\dot{S}=W+b d \frac{1}{\mathrm{~L}} u
$$

The control law $u$ thus obtained is:

$$
u=\left(\frac{L}{b d}\right)\left(-W-k_{1} \operatorname{sign}(S)-k_{2} S\right) .
$$

The designed control law, when subjected to a system for tracking purpose, resulted in undesirable chattering in the control input. For stability analysis, the Lyapunov function is defined as in Eq. (33) with its time derivative given in Eq. (34).

$$
\begin{gathered}
V=\frac{1}{2} s^{2}, \\
\dot{V}=s \dot{s} .
\end{gathered}
$$

Substituting Eq. (31) in Eq. (34), we obtain:

$$
\dot{V}=S\{W+b d(1 / L) u\} .
$$

Putting values in Eq. (35):

$$
\dot{V}=-k_{1}|S|-k_{2}|S|^{2} .
$$

Eq. (36) indicates that the derivative of the Lyapunov function is definite, which means that the system is asymptotically stable as long as both $k_{1}$ and $k_{2}>0$. The steady-state error can be calculated by taking Laplace transform of Eq. (30).

$$
E(s)=\frac{s^{3}+4 c s^{2}+6 c^{2} s+4 c^{3}}{s^{4}+4 c s^{3}+6 c^{2} s^{2}+4 s c^{3}+c^{4}},
$$

where the inverse Laplace transform is:

$$
e(t)=e(0)\left\{e^{c t}+\frac{c^{2} t^{2} e^{-c t}}{2}+\frac{c^{3} t^{3} e^{-c t}}{6}+c t e^{-c t}\right\} .
$$

The designed control input drives the steady-state error $e(t)$ to the sliding surface $S=0$ asymptotically i.e. $\lim e(t)=0$ with convergence rate given by Eq. (37) and remains there subject to positive gains of the controller [19].

\subsection{Integral Sliding Mode Control Technique}

To overcome the major drawbacks encountered in conventional SMC approach, the integral term can be included in SMC. The main idea behind ISMC is high-frequency switching gain, which is designed to force the state to achieve the integral sliding surface. Then, the integral action in the sliding manifold drives the states to the desired equilibrium point. It is an efficient control technique used to overcome several problems encountered in the SMC approach such as high-frequency chattering effect and its insensitivity property. It mitigates chattering and improves the robustness and accuracy of the control system while guaranteeing the nominal control performance. The 
dynamics of a flexible joint manipulator is explained in Eq. (19). The sliding manifold selected for the system is:

$$
s=\int e\left(c+\frac{d}{d t}\right)^{n},
$$

where $e=x_{1}-x_{d}$ is the error between the actual and desired outputs. $n$ is the relative degree of the system, $c$ is a constant known as design parameter, i.e. $c>0$. After expanding, we take,

$$
s=\int e c^{5}+5 c^{4} e+10 c^{3} \dot{e} .
$$

Taking the derivative of error variables:

$$
\begin{aligned}
e= & x_{1}-x_{d}, \quad \dot{e}=x_{2}-\dot{x}_{d}, \quad \ddot{e}=\dot{x}_{2}-\ddot{x}_{d}, \\
\dddot{e}= & -a \cos \left(x_{1}\right) \dot{x}_{1}-b \dot{x}_{1}+b \dot{x}_{3}-\dddot{x}_{d}, \\
\dddot{e}= & -a \cos \left(x_{1}\right) \dot{x}_{2}+a x_{2}^{2} \sin \left(x_{1}\right)-b \dot{x}_{2}+ \\
& +b\left(O\left(x_{1}-x_{3}\right)-\left(B / J_{2}\right) x_{4}+d x_{5}\right)+b f_{1}+\dddot{x}_{d} .
\end{aligned}
$$

For the sake of brevity, defining a symbol $Q$, i.e.

$$
\begin{aligned}
Q & =c^{5} e+5 c^{4} \dot{e}+10 C^{3}\left(\dot{x}_{2}-\ddot{x}_{d}\right)+ \\
& 10 c^{2}\left(-a \cos \left(x_{1}\right) x_{2}-b x_{2}+b x_{4}-\dddot{x}_{d}\right)+ \\
& 5 c\left(-a \cos \left(x_{1}\right) \dot{x}_{2}+a x_{2}^{2} \sin \left(x_{1}\right)-b \dot{x}_{2}+b \dot{x}_{4}-\dddot{x}_{d}\right)- \\
& a \cos \left(x_{1}\right) \ddot{x}_{2}+a x_{2} \dot{x}_{2} \sin \left(x_{1}\right)+2 a x_{2} \dot{x}_{2} \sin \left(x_{1}\right)+ \\
& a x_{2}^{3} \cos \left(x_{1}\right)-b \ddot{x}_{2}+b O\left(x_{2}-x_{4}\right)- \\
& \frac{b B}{J_{2}}\left(O\left(x_{1}-x_{3}\right)-\frac{B}{J_{2}} x_{4}+d x_{5}\right)+ \\
& b d\left(\frac{-R}{L} x_{5}-\frac{k_{b}}{L} x_{4}\right)-\dddot{x}_{d} \pi r^{2} .
\end{aligned}
$$

Putting the values and taking derivative of sliding surface, we obtain:

$$
\dot{s}=Q+\frac{b d}{L} u
$$

The control law $u$ thus obtained is:

$$
u=\frac{L}{b d}\left(-Q-k_{1} \operatorname{sign}(s)-k_{2} s\right) .
$$

For stability analysis, the Lyapunov function is given by:

$$
V=\frac{1}{2} s^{2}
$$

Taking time derivative of Eq. (42),

$$
\dot{V}=s \dot{s} .
$$

Substituting Eq. (40),

$$
\dot{V}=s\left\{Q+\frac{b d}{L} u\right\} \text {. }
$$

After putting values,

$$
\dot{V}=-k_{1}|s|-k_{2} s^{2} .
$$

ISMC ensures asymptotic stability in finite time. The steady state-error between the desired and the actual trajectory is calculated by considering the integral sliding manifold as:

$$
S=\int c^{5} e+5 c^{4} e+\dddot{e}+5 c \dddot{e}+10 c^{2} \ddot{e}+10 c^{3} \dot{e} .
$$

Assuming the condition $S=0$ and by taking the Laplace transform,

$$
E(s)=\left\{\frac{10 c^{3} s+10 c^{2} s^{2}+5 c s^{3}+s^{4}}{c^{5}+5 c^{4} s+10 c^{3} s^{2}+10 c^{2} s^{3}+5 c s^{4}+s^{5}}\right\} .
$$

Now taking inverse Laplace transform,

$$
e(t)=e(0) e^{-c t}\left\{1+\frac{c^{2} t^{2}}{2}+\frac{c^{3} t^{3}}{6}-\frac{c^{4} t^{4}}{6}+\mathrm{c} t\right\} \pi r^{2}
$$

Eq. (46) indicates the steady-state error with its convergence rate, which means that it approaches to zero in finite time, i.e. $\lim _{t \rightarrow \infty} e(t)=0$.

\section{SIMULATION AND EXPERIMENTAL RESULTS}

The performance and effectiveness of the designed controllers for desired tracking performance of flexible joint manipulators are verified through results gathered from simulations carried out in MATLAB/ Simulink and the custom-developed platform. The setup is a single-link flexible joint manipulator shown in Fig. 2.

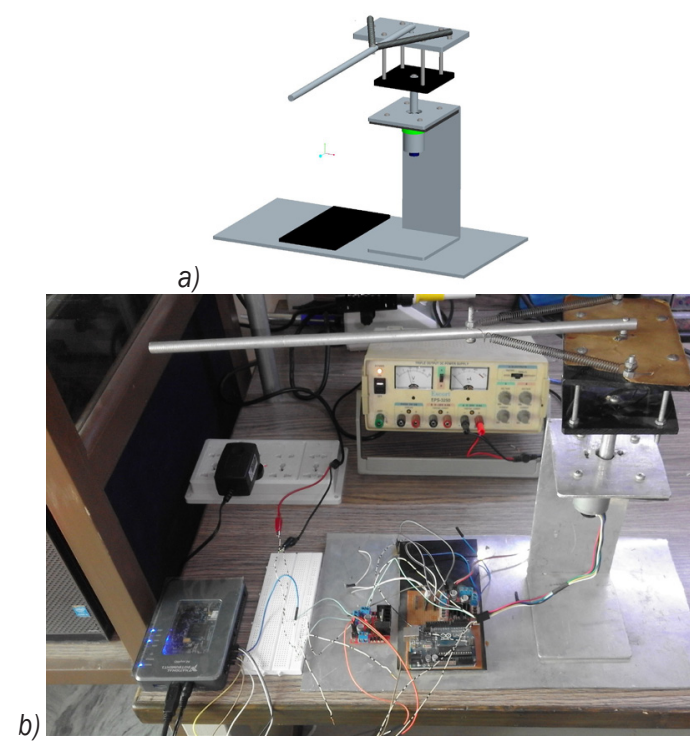

Fig. 2. Custom-developed experimental setup: a) $C A D$ model b) fabricated prototype 
The joint consists of an aluminium sheet tilted in such a way that a link is connected to a motor shaft through the sheet by two torsional springs. The actuator is a $24 \mathrm{~V}$ DC gear motor, actuated with pulse width modulated (PWM) signal, which converts the control effort into an amplified voltage using an H-Bridge L298 and MyRio-1900 controller kit. The DC gear motor drives the aluminium plate directly. Two quadrature encoders provide feedback of the angular positions corresponding to the motor and the link. The rotary encoder is attached to the flexible joint so as to provide the joint position independently of the motor's position. Real-time control implementation is carried out in LabVIEW connected with external hardware using a MyRio-1900 data acquisition device. Two types of trajectories are tested for tracking purposes: step and sinusoidal. Simulation and experimental results corresponding to both types of input are presented and discussed below.

\subsection{Step Tracking Using SMC and ISMC Laws}

In this case, the desired trajectory is a constant value function. It has been observed that SMC produces undesirable chattering phenomena in control input, which can make the system unstable at any time. The designed control law, when subjected to the system for tracking purposes, practically affects system's mechanical and electric parts. This adverse phenomenon can be eliminated using ISMC.

Fig. 3 presents simulation results in which the designed control law is tracking a unit step function of $60^{\circ}$ amplitude. The angular position shown in the figure is basically the position of the flexible joint. As evident from the results, the response of ISMC is better than SMC in terms of the steady-state error. However, the transient response of SMC shows good compliance with reference to the input.

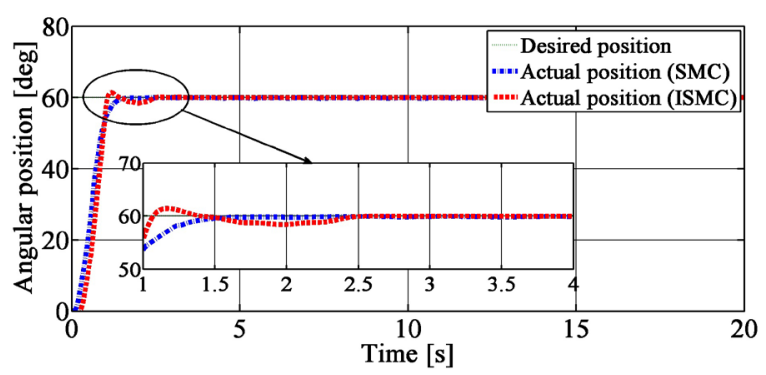

Fig. 3. Responses of step tracking in simulation by control laws based on SMC and ISMC

Fig. 4 shows the control input applied to the system in simulation. The results clearly show that the control input has undesirable chattering phenomenon in case of SMC, which can be harmful to the electrical and mechanical parts of the system.

Fig. 5 presents the results obtained from the experimental platform for tracking performance of the flexible joint manipulator using the SMC approach. The control input used for tracking purposes is also shown in the form of the percentage of the pulsewidth modulation (PWM) signal applied to the motor. The results clearly show that with the SMC approach, a flexible joint manipulator can reasonably track the desired trajectory, however, at the expense of undesirable chattering in the control input.

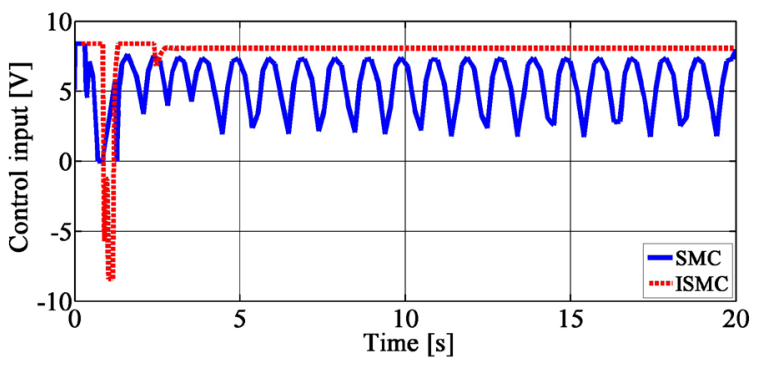

Fig. 4. Simulation results for the control input applied to SMC and ISMC (Step references)
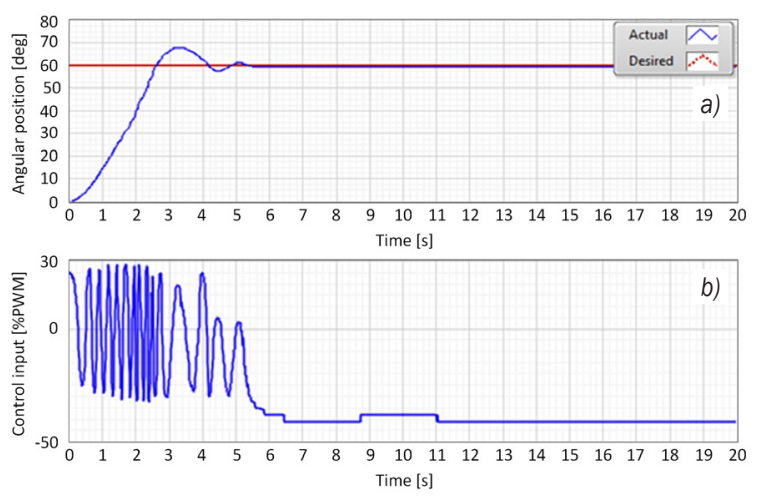

Fig. 5. Experimental results of a) trajectory tracking and b) the control effort using SMC

Fig. 6 shows the tracking error between actual trajectory and desired trajectory in case of SMC. The control law drives the steady-state error to zero within 5 seconds.

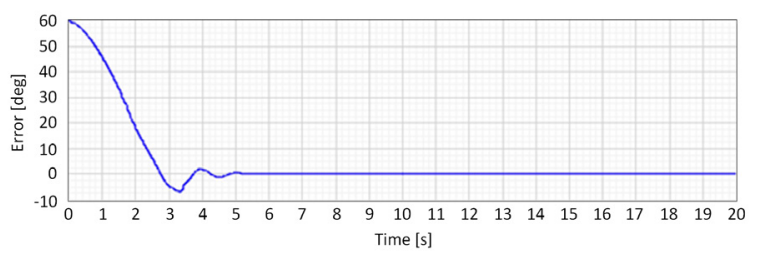

Fig. 6. Experimental results of steady-state error using SMC 
Fig. 7 shows experimental results of the ISMCbased law in which the tracking performance of flexible joint manipulator and the control input are presented. The results clearly show that when using an ISMC approach, the manipulator tracked the desired trajectory with reasonable transient as well as steady state performance.
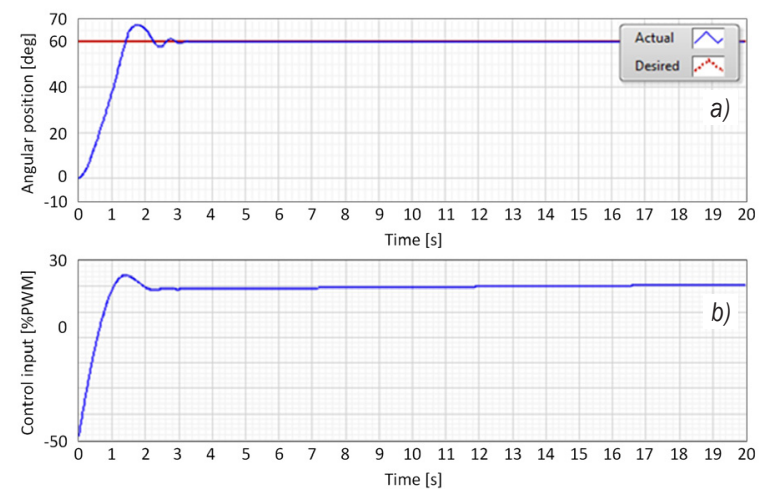

Fig. 7. Experimental results of the a) trajectory tracking and b) the control effort using ISMC

Fig. 8 shows trajectory tracking error between the actual and the desired positions, which is very small in comparison to SMC (see Fig. 6).

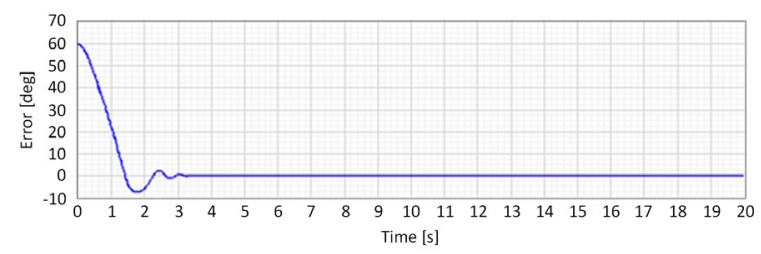

Fig. 8. Experimental results of steady-state error using ISMC

\subsection{Sinusoidal Input Tracking using SMC and ISMC}

In this case, the desired trajectory is a time-dependent sinusoidal function. The amplitude of the desired trajectory is taken as $60^{\circ}$ with the frequency set to as low as $0.0048 \mathrm{~Hz}$ for smooth tracking. In case of ISMC, results collected both from the simulation and the experimental platform reflect that chattering is reduced due to the continuous control action while preserving the robustness and accuracy of the controller to a high degree. This observation is consistent with the theoretical advantage of ISMC over the traditional SMC approach.

Fig. 9 presents simulation results in which the sinusoidal signal representing the angular position of the flexible joint manipulator serves as the reference for trajectory tracking. The results demonstrate that both the control laws exhibit good settling time and zero steady-state error.

Fig. 10 shows the simulated control input used to drive the flexible joint manipulator in order to track the desired trajectory. As is evident from the figure, in the case of SMC, the control input has undesirable chattering phenomena, which is an inherent property of SMC.

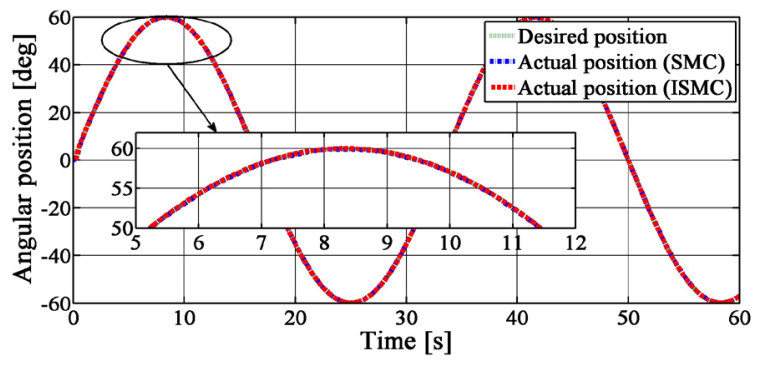

Fig. 9. Simulation results of sinusoidal input tracking for SMC and ISMC

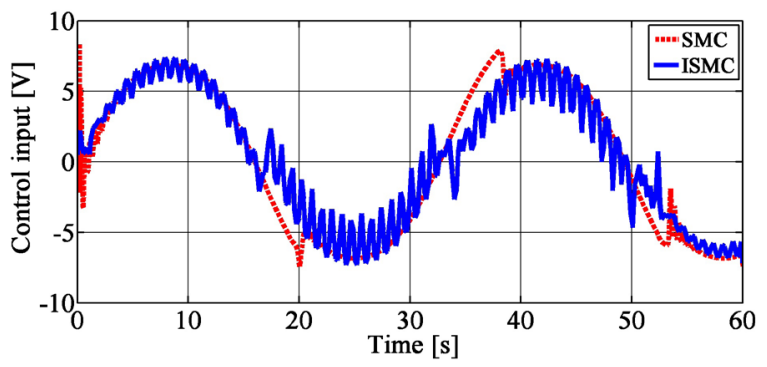

Fig. 10. Simulation results for the control input applied to SMC and ISMC (Sine reference)
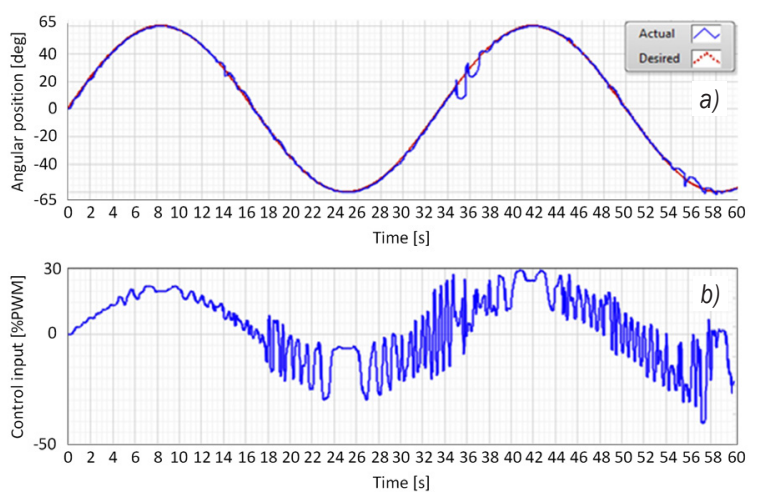

Fig. 11. Experimental results of a) trajectory tracking and b) control effort using SMC

Fig. 11 illustrates the desired trajectory tracking of a flexible joint manipulator obtained through experimental platform using SMC. This figure shows the tracking performance of the manipulator in real time along with control input, which was used for said purpose. These results confirm adequate tracking 
performance at the expense of undesirable chattering in the control input as observed in the simulation (see Fig. 10).

Fig. 12 shows errors in tracking between the actual trajectory and the desired trajectory when the manipulator is subjected to the SMC approach in real time.

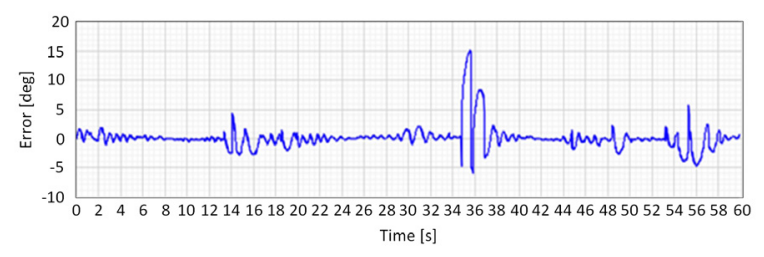

Fig. 12. Experimental result of steady-state error using SMC

Fig. 13 presents experimental results of the desired trajectory tracking of the manipulator based on the ISMC law. The control input used by the system for tracking purposes is also shown. Over performance of ISMC over SMC in terms of tracking performance is obvious while comparing Figs. 11 and 13. Furthermore, ISMC offers negligible steady-state error between the actual trajectory and the reference trajectory as depicted in Fig. 14.
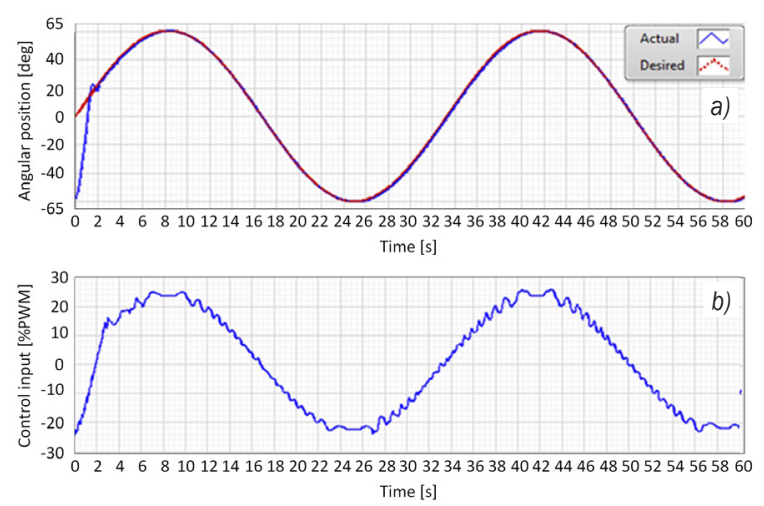

Fig. 13. Experimental results of a) trajectory tracking and b) control effort using ISMC

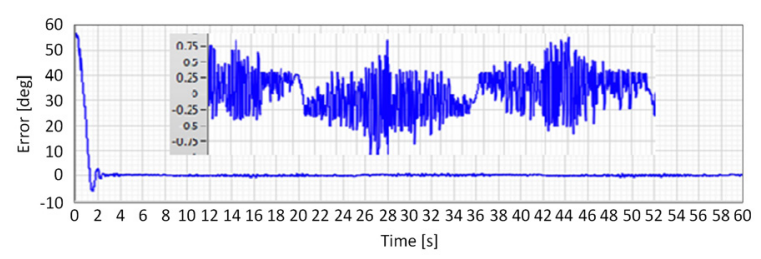

Fig. 14. Experimental results of steady-state error using ISMC

\section{CONCLUSION}

This research addresses the behaviour of a flexible joint manipulator including the actuator dynamic for nonlinear control approaches. The model of the manipulator has been derived using the EulerLagrange method, which is then used to study the effectiveness of the nonlinear control techniques for tracking performance. The nonlinear approaches under study include MSC and ISMC. A simulation has been conducted in MATLAB/Simulink while experimental validation of the designed control law has been carried out on a custom developed platform consisting of a single-link flexible joint manipulator. SMC approach resulted in chattering phenomena both in simulation and experimental results. This problem was eliminated by devising a control law based on ISMC, which also reduces steady-state error. Experimental results obtained in the present research can find enormous potential in application domains involving flexible robotic manipulators including but not limited to medical, space, and industrial automation.

\section{REFERENCES}

[1] Finžgar, M., Podržaj, P. (2017). Machine-vision-based humanoriented mobile robots: A review. Strojniški vestnik-Journal of Mechanical Engineering, vol. 63, no. 5, p. 331-348, DOl:10.5545/sv-jme.2017.4324.

[2] lqbal, J., Ajwad, S.A., Syed, Z.A., Abdul, A.K, Islam, R.U. (2016). Automating industrial tasks through mechatronic systems - A review of robotics in industrial perspective. Tehnički vjesnik Technical Gazette, vol. 23, no. 3, p. 917-924, D0l:10.17559/ TV-20140724220401.

[3] Ajwad, S.A., Asim, N., Islam, R.U, Iqbal, J. (2017). Role and review of educational robotic platforms in preparing engineers for industry. Maejo International Journal of Science and Technology, vol. 11, no. 1, p. 17-34.

[4] Salmasi, H., Fotouhi, R., Nikiforuk, P.N. (2009). Tip trajectory tracking of flexible-joint manipulators driven by harmonic drives. International Journal of Robotics \& Automation, vol. 24, no. 2, p. 147-157, DOl:10.2316/Journal.206.2009.2.206-3283.

[5] Dawson, D.M., Hu, J., Burg, T.C. (1998). Nonlinear Control of Electric Machinery, CRC Press, New York.

[6] Khan, O., Pervaiz, M., Ahmad, E., Iqbal, J. (2017). On the derivation of novel model and sophisticated control of flexible joint manipulator. Revue Roumaine des Sciences TechniquesSerie Electrotechnique et Energetique, vol. 62, no. 1, p. 103108.

[7] Ajwad, S.A., Ullah, M., Khelifa, B., Iqbal, J. (2014). A comprehensive state-of-the-art on control of industrial articulated robots. Journal of Balkan Tribological Association, vol. 20, no. 4, p. 499-521.

[8] Lozano, R., Brogliato, B., Egeland, O., Maschke, B. (2013). Dissipative Systems Analysis and Control: Theory and 
Applications. Springer Science \& Business Media, SpringerVerlag London.

[9] Ullah, M.I., Ajwad, S.A., Irfan, M., Iqbal, J. (2016). Non-linear control law for articulated serial manipulators: Simulation augmented with hardware implementation. Elektronika ir Elektrotechnika, vol. 22, no. 1, p. 3-7, D0l:10.5755/j01. eee.22.1.14094.

[10] Ajwad, S.A., Iqbal, J., Ullah, M.I., Mehmood, A. (2015). A systematic review of current and emergent manipulator control approaches. Frontiers of Mechanical Engineering, vol. 10, no. 2, p. 198-210, Dol:10.1007/s11465-015-0335-0.

[11] Iqbal, J., Ullah, M., Khan, S.G., Khelifa, B., Ćuković, S. (2017). Nonlinear control systems-A brief overview of historical and recent advances. Nonlinear Engineering, vol. 6, no. 4, p. 301312, Dol:10.1515/nleng-2016-0077.

[12] Ailon, A., Lozano, R., M.I. Gil' (2000). Iterative regulation of an electrically driven flexible-joint robot with model uncertainty. IEEE Transactions on Robotics and Automation, vol. 16, no. 6, p. 863-870, Dol:10.1109/70.897798.

[13] Chien, M.-C., Huang, A.-C. (2007). Adaptive control for flexiblejoint electrically driven robot with time-varying uncertainties. IEEE Transactions on Industrial Electronics, vol. 54, no. 2, p. 1032-1038, DOI:10.1109/TIE.2007.893054.

[14] Bang, J.S., Shim, H., Park, S.K., Seo, J. H. (2010). Robust tracking and vibration suppression for a two-inertia system by combining backstepping approach with disturbance observer. IEEE Transactions on Industrial Electronics, vol. 57, no. 9, p. 3197-3206, DOI:10.1109/TIE.2009.2038398.

[15] Liu, C., Cheah, C.C., Slotine, J.-J.E. (2008). Adaptive task-space regulation of rigid-link flexible-joint robots with uncertain kinematics. Automatica, vol. 44, no. 7, p. 1806-1814, D0l:10.1016/j.automatica.2007.10.039.

[16] Chang, Y.-C., Yen, H.-M. (2012). Robust tracking control for a class of electrically driven flexible-joint robots without velocity measurements. International Journal of Control, vol. 85, no. 2, p. 194-212, Dol:10.1080/00207179.2011.643241.

[17] Iqbal, J., Ullah, M.I., Khan, A.A., Irfan, M. (2015). Towards sophisticated control of robotic manipulators: An experimental study on a pseudo-industrial arm. Strojniški vestnik - Journal of Mechanical Engineering, vol. 61, no. 7-8, p. 465-470, DOl:10.5545/sv-jme.2015.2511.

[18] Ajwad, S.A., Adeel, M., Ullah, M.I., Iqbal, J. (2016). Optimal V/s Robust control: A study and comparison for articulated manipulator. Journal of the Balkan Tribological Association, vol. 22, no. 3, p. 2460-2466.

[19] Ajwad, S.A., Iqbal, J., Khan, A.A., Mehmood, A. (2015). Disturbance-observer-based robust control of a serial-link robotic manipulator using SMC and PBC techniques. Studies in Informatics and Control, vol. 24, no. 4, p. 401-408, DOI:10.24846/v24i4y201504. 